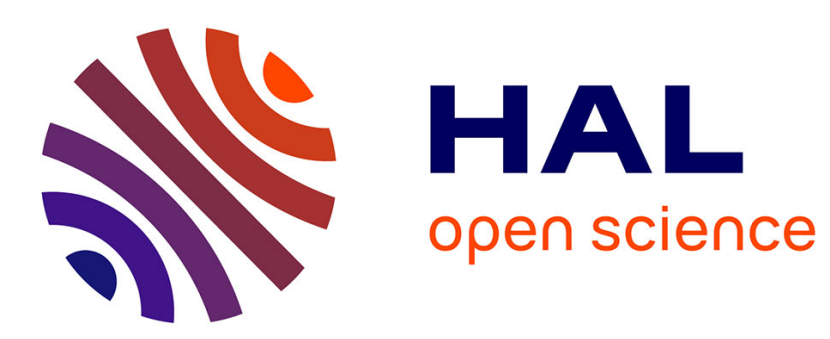

\title{
Limitation on the Method of Strained Coordinates for Vibrations with Weak Grazing Unilateral Contact
}

\author{
Stéphane Junca, Ly Tong
}

\section{To cite this version:}

Stéphane Junca, Ly Tong. Limitation on the Method of Strained Coordinates for Vibrations with Weak Grazing Unilateral Contact. Nonlinear Dynamics, 2015, 80, pp.197-207. 10.1007/s11071-0141860-9 . hal-01143612

\section{HAL Id: hal-01143612 https://hal.science/hal-01143612}

Submitted on 20 Apr 2015

HAL is a multi-disciplinary open access archive for the deposit and dissemination of scientific research documents, whether they are published or not. The documents may come from teaching and research institutions in France or abroad, or from public or private research centers.
L'archive ouverte pluridisciplinaire HAL, est destinée au dépôt et à la diffusion de documents scientifiques de niveau recherche, publiés ou non, émanant des établissements d'enseignement et de recherche français ou étrangers, des laboratoires publics ou privés. 


\title{
Limitation on the Method of Strained Coordinates for Vibrations with Weak Grazing Unilateral Contact
}

\author{
Stéphane JUNCA · Ly TONG
}

the date of receipt and acceptance should be inserted later

\begin{abstract}
The NNM (Nonlinear normal modes) have recently been investigated with the method of strained coordinates for spring mass models with some springs with piecewise linear stiffness. The $\mathrm{N}$ d.o.f. case and the mathematical validity of the method for large time were rigorously proved. The time validity is related to the nature of contact. For grazing contact, this method and also the multiscale expansion lose nonlinear features. For a small piecewise linear stiffness, we show that this method is less precise for a weak unilateral grazing contact. Thus, the validity of the asymptotic expansion for large time can not be improved and the method has to be modified.
\end{abstract}

Keywords vibration; piecewise-linear; grazing contact; perturbation; asymptotic analysis; LindstedtPoincaré method

Mathematics Subject Classification (2000) 34E10, (70K75,34E13)

\section{Introduction}

The NNM (Nonlinear normal modes $[1,2]$ ) have been investigated with the method of strained coordinates by Vestroni, Luongo, Paolone in [3] for a two d.o.f. spring mass models with some springs with piecewise linear stiffness. Then the $\mathrm{N}$ d.o.f case has been studied in [4]. The method of strained coordinates, also

Stéphane Junca

Mathematics Laboratory JAD and Team COFFEE INRIA, Université de Nice Sophia-Antipolis, junca@unice.fr

Ly TONG

Royal University of Phnom Penh,

tongkhmer2008@yahoo.com named Lindstedt-Poincaré method, is usfeul to compute periodic solution for weakly nonlinear problem $[5$, $6]$. The mathematical validation for smooth forcing is well knonwn, see for instance [7]. This method has been used for less smooth forcing: piecewise linear force, by Vestroni \& al. in [3] and independently in [8]. Numerical experiments have been investigated in [3] and also in [4]. The mathematical validity is obtained in [4]. But the accuracy is less precise for the grazing contact case. It is well known that the grazing contact yields to fractional power expansions instead of integer power expansions, for instance see [9] for bifurcations and [10] for contacts (Signorini problem) and references therein. In this paper, we also show on a simple example that this loss of precision is due to the method of strained coordinates in a presence of a grazing contact. The method of strained coordinates is useful to look for Nonlinear Normal Modes for systems with $N$ d.o.f. (degree of freedom), $N>1$. To explain the limitation of the method, we focus on a one d.o.f. problem, typically:

$\ddot{u}+u+\varepsilon f(u)=0, u(0)=u_{0}, \dot{u}(0)=0, f^{\prime}>0$.

In this case, the solution is always periodic. The problem is to find a good approximation of the solution for $0<\varepsilon \ll 1$. A simple approximation by the linear problem $\varepsilon=0$ is good enough to have an approximation of order 0 of the solution and the period. But the nonlinear effect is missed. An expansion of the solution $u_{\varepsilon}=u_{0}+\varepsilon u_{1}+\cdots$ is good enough for a finite time but not enough for large time. It is well known that the nonlinear effect appears for large time $[5,6,7,11]$. A good idea is to expand the exact period $T(\varepsilon)$

$$
\begin{aligned}
T(\varepsilon) & =T_{0}+\varepsilon T_{1}+\varepsilon^{2} T_{2}+\mathcal{O}\left(\varepsilon^{3}\right), \\
& =T_{\varepsilon}+\mathcal{O}\left(\varepsilon^{3}\right),
\end{aligned}
$$


or, equivalently, the pulsation:

$$
\begin{aligned}
\omega(\varepsilon) & =\frac{2 \pi}{T(\varepsilon)} \\
& =\omega_{0}+\varepsilon \omega_{1}+\varepsilon^{2} \omega_{2}+\mathcal{O}\left(\varepsilon^{3}\right), \\
& =\omega_{\varepsilon}+\mathcal{O}\left(\varepsilon^{3}\right) .
\end{aligned}
$$

Notice the presence of the remainder in the equality:

$$
\omega_{\varepsilon}=\frac{2 \pi}{T_{\varepsilon}}+\mathcal{O}\left(\varepsilon^{3}\right)
$$

The method is usually presented with the pulsation. We choose to present the method with the period. Indeed, we will compare the period given by the method with an exact formula for a one d.o.f. model. For a one d.o.f. model, it is well known that the period can be computed by the mechanical energy. Thus we can study the accuracy of the method by comparing the period obtained by the Lindstedt-Poincaré method with its exact value obtained by the energy.

For the method of strained coordinates, we have to find the approximation of the solution in the rescaled time:

$$
\begin{aligned}
s & =\omega_{\varepsilon} t, \\
v_{\varepsilon}\left(\omega_{\varepsilon} t\right) & =u_{\varepsilon}(t) .
\end{aligned}
$$

For large time: $t \in\left[0, t_{\max }\right]$, an expansion of $v_{\varepsilon}$ is performed in the new time $s$ :

$v_{\varepsilon}(s)=v_{0}(s)+\varepsilon v_{1}(s)+\mathcal{O}\left(\varepsilon^{2}\right)$.

For smooth nonlinearity: $f \in C^{2}(\mathbb{R}, \mathbb{R})$, we have the rigorous approximation proven in $[5,6,7]$ :

$$
\begin{aligned}
u_{\varepsilon}(t) & =v_{0}\left(\omega_{\varepsilon} t\right)+\varepsilon v_{1}\left(\omega_{\varepsilon} t\right)+\mathcal{O}\left(\varepsilon^{2}\right), \\
\text { for } \quad t & \in\left[0, t_{\max }\right], \\
t_{\max } & \sim \frac{\text { constant }}{\varepsilon} .
\end{aligned}
$$

Indeed, the Lindstedt-Poincaré method is a particular case of the method of multiple scale expansions. Here, it is a double scale expansion with two times $(t, \varepsilon t)$. Nevertheless, with the Lindstedt-Poincaré method to look for periodic solutions, computations are simpler and can be used for less smooth case [3,4]. Surprisingly the precision for piecewise linear restoring function $f$ is the same as the $C^{2}$ case, except for grazing contact. Indeed, for $N$ d.o.f. and for the grazing contact, it is proven in [4] that this asymptotic expansion is valid at least for

$t_{\max } \leq \frac{\text { constant }}{\sqrt{\varepsilon}}$.

We will prove the optimality of this estimation on a simple one d.o.f. model. This is due for a lack of precision of the period. Indeed, the right expansion of the period for the grazing contact with piecewise linear force $f$ becomes:

$T(\varepsilon)=T_{0}+\varepsilon^{2.5} T_{2}^{*}+\mathcal{O}\left(\varepsilon^{3.5}\right)=T_{\varepsilon}^{*}+\mathcal{O}\left(\varepsilon^{3.5}\right)$.

As we will see, the classic method of strained coordinates is not able to compute $T_{2}^{*}$ and then $T_{\varepsilon}^{*}$. Thus the method only gives the less precise estimate:

$T(\varepsilon)=T_{0}+\mathcal{O}\left(\varepsilon^{2.5}\right)$.

This is the reason why $t_{\max }$ looses a fractional power of $\varepsilon$ and the Lindsted-Poincaré method is reduced to the crude approximation consisting to take $\varepsilon=0$ in equation (1). The aim of the paper is to prove rigorously this loss of precision in this case. Thus, the method of strained coordinates has to be modified to study nonlinear effects of grazing contact with piecewise linear forces.

The paper is organised as follows. In Section 2, the mechanical model is shortly presented. Section 3 gives the first mathematical results on the dimensionless model: the energy and the exact period. All computations are performed on the dimensionless model for the rest of the paper. In Section 4, the Lindstedt-Poincaré method is used for a grazing contact case. Section 5 is devoted to expand rigorously the period with respect to $\varepsilon$ for the grazing contact case. The new result is that the power of $\varepsilon$ involved in the period expansion are not integers but fractional numbers. This expansion is used to obtain the precision of the Lindstedt-Poincaré method in Section 6. Some numerical experiments are drawn in Section 7. Finally, we give some conclusions and some possible improvements in Section 8.

\section{The model}

We consider a one degree of freedom spring-mass system, Figure 1: one spring is linear and attached to the mass and to a rigid wall, the second is still linear attached to a rigid wall but has only a unilateral contact with the mass only when $U>a$; this is to be considered as a simplified model of a damaged spring. We assume $a>0$, i.e. at rest the "damaged" spring is on the right to the mass. To modeling this unilateral contact we use the function:

$U_{+}=\max (0, U)= \begin{cases}U & \text { if } U>0 \\ 0 & \text { else }\end{cases}$

This is a Lipschitz function but non differentiable at $U=0$. Indeed, $U \mapsto U_{+}$is a piecewise linear function. The force acting on the mass is $-\left(k_{1} U+k_{2}[U-a]_{+}\right)$ where $U$ is the displacement of the mass $m, k_{1}$ is the rigidity of the undamaged spring and $k_{2}$ is the rigidity 
of the "damaged" unilateral spring. We consider the equation:

$m \ddot{U}+k_{1} U+k_{2}[U-a]_{+}=0$.

We are interested by the grazing contact: $U \simeq a$. Thus
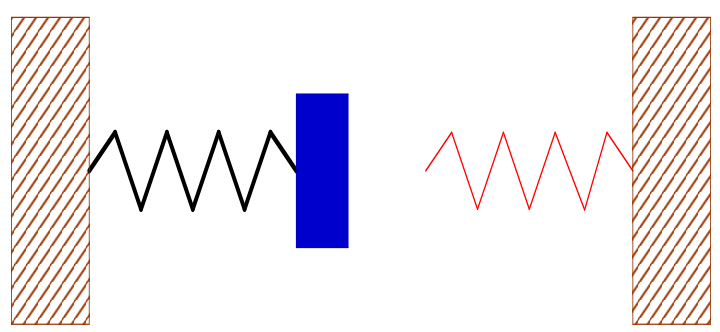

Fig. 1 One mass and two springs, on the right it has only a weak unilateral contact.

we study ODE (3) with such typical initial data:

$U(0) \simeq a, \quad \dot{U}(0)=0$.

The linear pulsation is $\omega_{0}^{2}=k_{1} / m$. If $U(0) \leq a$, then the solution is simply the solution to the linear ODE: $U(t)=a \cos \left(\omega_{0} t\right)$. Thus, the nonlinear effect only appears when $U(0)>a$.

Our study is a perturbation analysis, so we also assume that the "damaged" spring is weak compared to the other spring:

$k_{1} \gg k_{2}$.

We fix the notations by considering the dimensionless problem below.

\section{The dimensionless model}

Condition (5) is rewritten

$0<\varepsilon=\frac{k_{2}}{k_{1}} \ll 1$.

We rescale the displacement : $U=a u$ and the time with the new time $\omega_{0} t$. Equation (3) becomes:

$\ddot{u}+u+\varepsilon[u-1]_{+}=0$.

We are interested by the following initial data:

$u(0)=u_{0}=1+h_{0}>1, \quad \dot{u}(0)=0$.

\subsection{The energy}

The energy is the key tool to study ODE (7). We choose the slightly modified energy:

$E=E(u, \dot{u})=\dot{u}^{2}+F(u)=\dot{u}^{2}+u^{2}+\varepsilon\left([u-1]_{+}\right)^{2}$.

Notice that the mechanical energy is usually $E / 2$ and the potential energy is $F / 2$. We skip the constant 2 to simplify the notation. For any solution of (7) with initial data (8), we have the conservation of the energy: $\dot{E}=0$, i.e.

$E(u(t), \dot{u}(t))=E\left(u_{0}, 0\right)=E_{0}=F\left(u_{0}\right)=u_{0}^{2}+\varepsilon h_{0}^{2}$.

Therefore, in the phase space $(u, \dot{u})$, the level sets of $E(u, \dot{u})$ will be made of two pieces of ellipse symmetric with respect to the horizontal $u$ axis since $E(u,-\dot{u})=$ $E(u, \dot{u})$. Indeed, for $u<1$ the level set is a piece of a circle centered at the origin, and for $u>1$ is a piece of an ellipse. In Figure 2, we show the circle and the ellipse with the initial data $u_{0}=2.5$ and $\varepsilon=1.5$, in such a way the tangency and the difference between the two trajectories can be clearly appreciated (see also for example Figure 2 of [3]).

More precisely, $u_{-} \leq u(t) \leq u_{0}$ for all $t$ where $u_{-}$ satisfies:

$u_{-}<0<u_{0} \quad$ and $\quad F\left(u_{-}\right)=F\left(u_{0}\right)$.

A simple computations yields $-u_{-}=u_{0}+\varepsilon h_{0}^{2} / 2+$ $\mathcal{O}\left(\varepsilon h_{0}^{3}\right)$ for $u_{0} \sim 1$ and $u_{0}>1$, i.e. $h_{0} \sim 0$ and $h_{0}>0$.

Any solution $u(t)$ is confined to a closed level curve of $E(u, \dot{u})$. There is no equilibrium on this curve since $E>1>0$, so the solution is necessarily a periodic function of $t$.

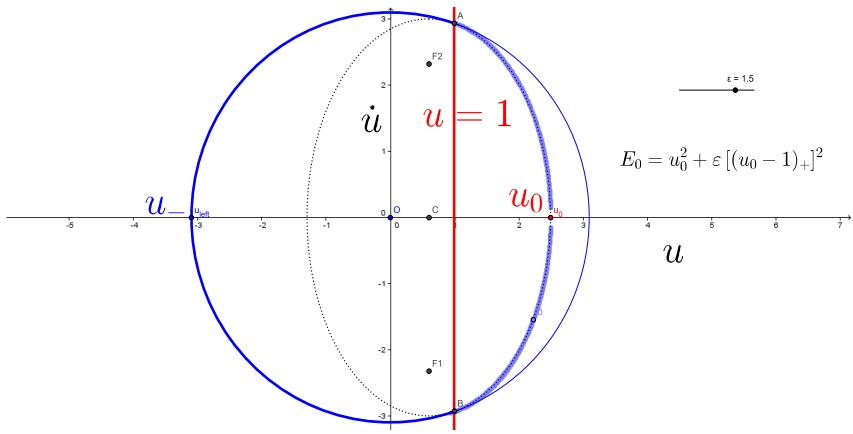

Fig. 2 Phase space $(u, \dot{u})$, energy level $E(u, \dot{u})=E_{0}$, for $u<1$ a piece of circle in blue, for $u>1$ a little piece of an ellipse in black dotted line. 


\subsection{The exact period}

The exact period is computed exactly.

$$
\begin{array}{r}
T=\left\{\begin{array}{r}
2 \pi-2 \arccos \left(\left|u_{-}\right|^{-1}\right) \\
+2 \sqrt{\eta} \arccos \left(\frac{\eta}{h_{0}+\eta}\right)
\end{array}\right. \\
u_{-}=-\sqrt{E_{0}}, \quad \eta=\frac{1}{1+\varepsilon} .
\end{array}
$$

When $\varepsilon=0$ and $h_{0}=0$ we recover the period $2 \pi$ of the linear ODE. For a one dof problem the classical but long computations using the energy is postponed in Appendix 1.

\section{The Lindstedt-Poincaré method}

We introduce briefly the method of strained coordinates, also named the Lindstedt-Poincaré method. When we look for a periodic solution of (7), in general the period is an unknown. We have already computed the exact period $T$ in (11) since this is only a one d.o.f. problem. For $N$ d.o.f. problem the period is an unknown and the Lindstedt-Poincaré method is an asymptotic method to compute an approximation of the period $[6$, $7,11,3,4]$.

The basic idea is to change the time to seek a 1periodic solution. Let $\omega(\varepsilon)$ be the exact pulsation:

$\omega(\varepsilon)=2 \pi / T$. Notice that for the linear problem: $\varepsilon=0$, we have simply $\omega(0)=1$. Let $\omega_{\varepsilon}$ be an approximation of the exact angular frequency $\omega(\varepsilon)$ which is smooth with respect to $\varepsilon$ as we can see in formula (11).

$\omega(\varepsilon)=\omega_{\varepsilon}+\mathrm{O}\left(\varepsilon^{3}\right)$.

Let us define the new time

$s=\omega_{\varepsilon} t$,

and rewrite equation (7) with $v_{\varepsilon}(s)=u_{\varepsilon}(t)$ :

$$
v_{\varepsilon}(s)=v_{\varepsilon}\left(\omega_{\varepsilon} t\right)=u_{\varepsilon}(t),
$$

$\omega_{\varepsilon}^{2} v_{\varepsilon}^{\prime \prime}(s)+v_{\varepsilon}(s)+\varepsilon\left(v_{\varepsilon}(s)-1\right)_{+}=0$.

$v_{\varepsilon}$ is subjected to the following initial conditions:

$v_{\varepsilon}(0)=1+h_{0}, v_{\varepsilon}^{\prime}(0)=0$.

In the new time $s$, we use the following ansatz

$\omega_{\varepsilon}=1+\varepsilon \omega_{1}+\varepsilon^{2} \omega_{2}$,

$v_{\varepsilon}(s)=v_{0}(s)+\varepsilon v_{1}(s)+\varepsilon^{2} r_{\varepsilon}(s)$.

$v_{0}$ represents the linear behavior and the other terms, $\omega_{1}, v_{1}, \omega_{2}$ and $r_{\varepsilon}$ are related to the nonlinear behavior.

\subsection{The grazing contact case:}

At this stage we take a key assumption on the initial data modeling the grazing contact. As said previously, we want to observe a grazing contact. For $u_{0} \leq 1$, the solution is only $v_{\varepsilon}(s)=u_{0} \cos (s)$ and we miss the nonlinear behavior. Clearly $u_{0}=1$ is the grazing contact case, but it is to simple for this one d.o.f. example. For a $\mathrm{N}$ d.o.f. system it is more complicate, see [4]. More precisely, in the coordinates formed by the eigenvectors of the linear equation, $u=\left(u_{1}, \cdots, u_{N}\right)$ an the initial data to find the NNM associated with the first linear mode is

$u_{1}(0)=a_{0}+\varepsilon a_{1}, \dot{u}_{1}(0)=0$,
$u_{k}(0)=0+\varepsilon a_{k}, \dot{u}_{k}(0)=0, k=2, \cdots, N$,

where $a_{0}$ is fixed and $a_{1}, a_{2}, \cdots, a_{N}$ are unknown. Thus, for $N$ d.o.f., the right expansion for $u_{1}(0)$ is dependent of $\varepsilon$ :

$$
u_{1}(0)=a_{0}+\varepsilon a_{1} .
$$

Nevertheless, we want to take the advantage of the 1 d.o.f. example which has an exact formula for the period and so for $\omega(\varepsilon)$. Thus, we have to take $u_{0}=1+h_{0}>1$. But, for $\varepsilon<<h_{0}$, the behavior is well approached by the method of strained coordinates, see [3] for good numerical approximations and see [4] for a mathematical validation. The good compromise is to take $h_{0} \sim \varepsilon$. At the first order, the linear approximation grazes the contact at $u=1$ and there is a small nonlinear interaction with the contact. Thus, we assume for all the sequel $h_{0}=\varepsilon$ and then:

$$
u(0)=1+\varepsilon .
$$

Now we can continue to use the Lindstedt-Poincaré method. Notice that $\omega_{1}$ and $\omega_{2}$ are unknown. Since

$\omega_{\varepsilon}^{2}=1+\varepsilon \alpha_{1}+\varepsilon^{2} \alpha_{2}+\mathcal{O}\left(\varepsilon^{3}\right)$,

$\alpha_{1}=2 \omega_{1}$,

$\alpha_{2}=\omega_{1}^{2}+2 \omega_{2}$,

we have to find $\alpha_{1}$ and $\alpha_{2}$.

We will also use the following expansion,

$(u+\varepsilon v)_{+}=u_{+}+\varepsilon H(u) v+\varepsilon \chi_{\varepsilon}(u, v)$

where $H($.$) is the Heaviside function:$

$$
H(u)= \begin{cases}1 & \text { if } u>0 \\ 0 & \text { else }\end{cases}
$$

Since $H($.$) is not differentiable at u=0$, the remainder $\varepsilon \chi_{\varepsilon}(u, v)$ is not the classical Taylor's remainder. This lack of smoothness is a problem to validate mathematically the Lindstedt-Poincaré method. The remainder term is mathematically rigorously justified in [4]. 
Now, replacing ansatz (15) in (14) we obtain differential equations and initial data for $v_{0}, v_{1}, r_{\varepsilon}$ :

$$
\begin{aligned}
L\left(v_{0}\right)= & -\left(v^{\prime \prime}+v\right)=0, \\
& v_{0}(0)=1, \quad v_{0}^{\prime}(0)=0, \\
L\left(v_{1}\right)= & \left(v_{0}-1\right)_{+}+\alpha_{1} v_{0}^{\prime \prime}, \\
& v_{1}(0)=1, \quad v_{1}^{\prime}(0)=0, \\
L\left(r_{\varepsilon}\right)= & H\left(v_{0}-1\right) v_{1}+\alpha_{2} v_{0}^{\prime \prime}+\alpha_{1} v_{1}^{\prime \prime}+R_{\varepsilon}(s), \\
& r_{\varepsilon}(0)=0, \quad r_{\varepsilon}^{\prime}(0)=0 .
\end{aligned}
$$

We have $v_{0}(s)=\cos (s)$ so $\left(v_{0}-1\right)_{+}=0$ and $H\left(v_{0}-1\right)=$ 0 , so previous equations are simplified:

$$
\begin{aligned}
L\left(v_{1}\right)= & \alpha_{1} v_{0}^{\prime \prime}, \\
& v_{1}(0)=1, \quad v_{1}^{\prime}(0)=0, \\
L\left(r_{\varepsilon}\right)= & \alpha_{2} v_{0}^{\prime \prime}+\alpha_{1} v_{1}^{\prime \prime}+R_{\varepsilon}(s), \\
& r_{\varepsilon}(0)=0, \quad r_{\varepsilon}^{\prime}(0)=0 .
\end{aligned}
$$

A key point in the method of strained coordinates is to look for a periodic solution, so to avoid resonance with the right hand side. To keep bounded $v_{1}$ and $r_{\varepsilon}$ for large time we chose carefully $\alpha_{1}$ for $v_{1}$ and $\alpha_{2}$ for $r_{\varepsilon}$. For this purpose, we avoid resonant or secular term in the right-hand-side of Equation (20) only when $\alpha_{1}=0$. Thus $v_{1}(s)=\varepsilon \cos (s)$.

In the same way, we have to take $\alpha_{2}=0$. The approximations is then:

$$
\begin{aligned}
\omega_{\varepsilon} & =1, \\
v_{0}(s)+\varepsilon v_{1}(s) & =(1+\varepsilon) \cos (s) .
\end{aligned}
$$

\subsubsection{What is the accuracy of this approximation?}

Assumptions (15) suggest the following precision:

$$
\begin{aligned}
\omega(\varepsilon) & =1+\mathcal{O}\left(\varepsilon^{3}\right), \\
T(\varepsilon) & =1+\mathcal{O}\left(\varepsilon^{3}\right), \\
u_{\varepsilon}(t) & =(1+\varepsilon) \cos (t)+\mathcal{O}\left(\varepsilon^{2}\right), \\
\text { for } \quad t & \leq \frac{\text { constant }}{\varepsilon} .
\end{aligned}
$$

But it is not the right precision as we will see below. Before the rigorous proof in following sections we give some hints to correct the error terms $\mathcal{O}\left(\varepsilon^{3}\right)$ and the time validity $t \leq \frac{\text { constant }}{\varepsilon}$ in $(23)$. The right precision is found in (24) and more precised in next sections.

\subsubsection{Heuristic argument on the precision}

The Lindstedt-Poincaré method miss the nonlinear behavior of the solution of the nonlinear ODE (7) since the method gives only the solution of the linearized part of the ODE, namely $v(t)=(1+\varepsilon) \cos (t)$ which is the solution of

$\ddot{v}+v=0, v(0)=u(0)=(1+\varepsilon), \dot{v}(0)=\dot{u}(0)=0$.
The nonlinear term $\varepsilon[v-1]_{+}$is missing in the previous ODE.

Indeed, the lack of precision is explicitely given by the expansion of the exact period. The period founded by the Lindstedt-Poincaré method is $T_{\varepsilon}=2 \pi$ since $\omega_{\varepsilon}=1$. There is no dependence with respect to $\varepsilon$. Before, making more precise computations, we give a hint of the error size. Notice that the contact occurs at the maximum of $v_{\varepsilon}$, when $1<v_{\varepsilon}$. We expect that the maximum of $v_{\varepsilon}$ is of order $1+\varepsilon$ so the nonlinear effect is not taken account when $v_{\varepsilon}(s)$ stays approximatively in the interval $] 1,1+\varepsilon\left[\right.$ which size $\simeq \varepsilon$. At the maximum, $v_{\varepsilon}$ behaves like a parabola, so the time $s$ are in an interval with a length of order $\sqrt{\varepsilon}$, see Figure 2. Thus we expect to have an error on $T(\varepsilon)$ of order:

$$
\sqrt{\varepsilon} \times \varepsilon\left(v_{\varepsilon}-1\right)_{+} \simeq \varepsilon^{2.5}
$$

which is bigger than the usual $\mathcal{O}\left(\varepsilon^{3}\right)$ for the method. We show below that the period is not so well computed. The error is exactly $\simeq \varepsilon^{2.5}$. This error on the period of order $\sqrt{\varepsilon}$ spoils the validity of asymptotic expansions for too much large time. Indeed, we have to correct (23) by:

$$
\begin{aligned}
\omega(\varepsilon) & =1+\mathcal{O}\left(\varepsilon^{2.5}\right), \\
T(\varepsilon) & =1+\mathcal{O}\left(\varepsilon^{2.5}\right) \\
u_{\varepsilon}(t) & =(1+\varepsilon) \cos (t)+\mathcal{O}\left(\varepsilon^{2}\right), \\
\text { for } \quad t & \leq \frac{\text { constant }}{\sqrt{\varepsilon}} .
\end{aligned}
$$

\section{The period for the grazing contact}

In this section we obtain (26): the asymptotic expansion of the period $T(\varepsilon)$. That is to say the period for the solution of ODE (7) with initial data

$u(0)=1+\varepsilon, \quad \dot{u}(0)=0$.

The Lindstedt-Poincaré method is well known to obtain an approximate period $T_{\varepsilon}$ with a precision of order 3 for smooth forcing: $T(\varepsilon)=T_{\varepsilon}+\mathcal{O}\left(\varepsilon^{3}\right)$. In the piecewise linear case with a grazing contact we loose a precision of order $\sqrt{\varepsilon}$. Indeed, we show in Appendix 2 below that the exact period admits the following expansion.

$T(\varepsilon)=2 \pi-\frac{7}{6} \sqrt{2} \varepsilon^{2.5}+\mathcal{O}\left(\varepsilon^{3.5}\right)$.

Since the Lindstedt-Poincaré method only gives $T_{\varepsilon}=$ $2 \pi$ we have proved that:

$T(\varepsilon)=T_{\varepsilon}+\mathcal{O}\left(\varepsilon^{2.5}\right)$.

Notice that the remainder is not smaller than $\mathcal{O}\left(\varepsilon^{2.5}\right)$ since from (26) we have:

$$
T(\varepsilon)-T_{\varepsilon} \sim-\frac{7}{6} \sqrt{2} \varepsilon^{2.5} .
$$




\section{Precision of the Lindstedt-Poincaré method}

The usual expected precision for the method of strained coordinates is given in (23) . But we have rigorously shown in a previous section that the period is only approximated by the linear period with a bigger remainder: $T(\varepsilon)=2 \pi+\mathcal{O}\left(\varepsilon^{2.5}\right)$ and then

$\omega(\varepsilon)=1+\mathcal{O}\left(\varepsilon^{2.5}\right)$.

We now explain the consequence of this loss of precision on the periodic solution and we justify the right expansion (24). First, for the exact solution $u_{\varepsilon}(t)$ of $(7), v_{\varepsilon}(s)$ of $(14)$ and the exact pulsation $\omega(\varepsilon)$ we have the equaltiy without anny remainder:

$u_{\varepsilon}(t)=v_{\varepsilon}(\omega(\varepsilon) t)$

Notice that for $0 \leq \varepsilon \leq 1, u_{\varepsilon}$ is uniformly bounded in $C^{1}$ thanks to the energy (9). Then $v_{\varepsilon}$ is also uniformly bounded in $C^{1}$. Now, replacing the exact pulsation $\omega(\varepsilon)$ with equality $(27)$ we have:

$$
\begin{aligned}
u_{\varepsilon}(t) & =v_{\varepsilon}\left(\left[\omega_{\varepsilon}+\mathcal{O}\left(\varepsilon^{2.5}\right)\right] t\right), \\
& \left.=v_{\varepsilon}\left(\omega_{\varepsilon} t\right)\right)+\mathcal{O}\left(\varepsilon^{2.5}\right) t .
\end{aligned}
$$

Thus, to keep a remainder of order 2 we have to take $t \leq t_{\max }=\frac{\text { constant }}{\sqrt{\varepsilon}}$.

Furthermore, we do not know the exact solution $v_{\varepsilon}(s)=$ $v_{0}(s)+\varepsilon v_{1}(s)+\varepsilon^{2} r_{\varepsilon}(s)$. Fortunately, the remainder $r^{\varepsilon}(s)$ is bounded in [4] for $t \leq t_{\max }$. Then $u_{\varepsilon}(t)=$ $\left.\left.v_{0}\left(\omega_{\varepsilon} t\right)\right)+\varepsilon v_{1}\left(\omega_{\varepsilon} t\right)\right)+\mathcal{O}\left(\varepsilon^{2.5}\right)$ for $t \leq t_{\max }$ which proves $(24)$.

\section{Numerical experiments}

In this section we compare the exact solution computed in Appendix 3, the strained coordinates approximation which is here the linear approximation $(1+\varepsilon) \cos (t)$ and the improved approximation using the asymptotic expansion of the period $(1+\varepsilon) \cos \left(\omega_{\varepsilon}^{*} t\right)$ and the linear approximation. From the expansion of $T(\varepsilon)$ we take $\omega_{\varepsilon}^{*}=1+\frac{7}{6 \sqrt{2} \pi} \varepsilon^{2.5}$.

The exact solution is given by the black line. The strained coordinates approximation is represented by red crosses. The improved solution is represented by blue circles.

For the time validity $\frac{1}{\sqrt{\varepsilon}}$ justified in [4] we have on the time interval $\left[\frac{1}{\sqrt{\varepsilon}}, \frac{1}{\sqrt{\varepsilon}}+T(\varepsilon)\right]$ the following results in Figures 3,4,5.

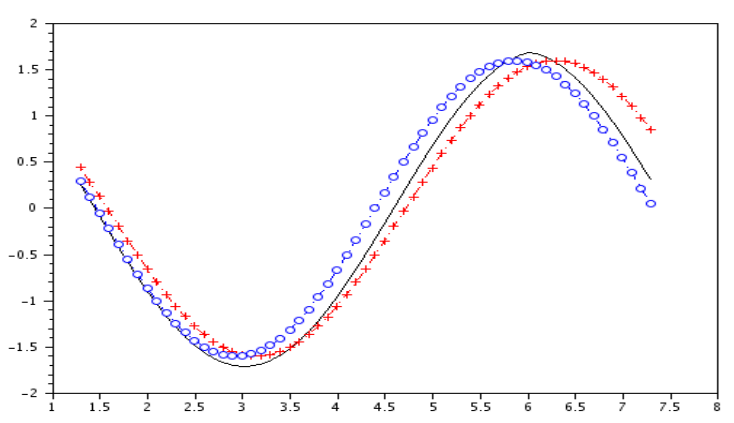

Fig. $3 \varepsilon=0.6$, time interval $\left[\varepsilon^{-1 / 2}, \varepsilon^{-1 / 2}+T(\varepsilon)\right]$.

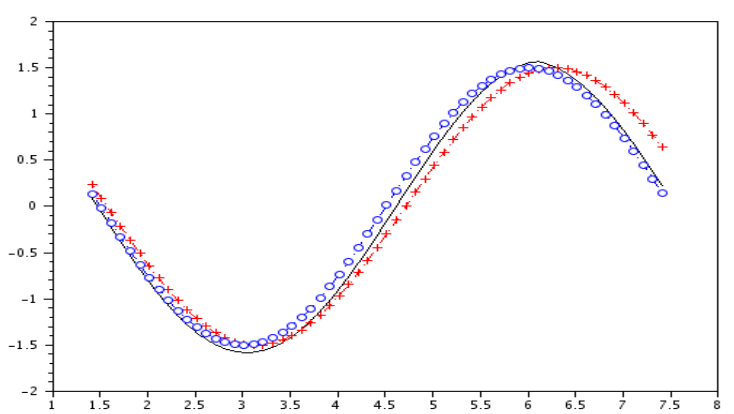

Fig. $4 \varepsilon=0.5$, time interval $\left[\varepsilon^{-1 / 2}, \varepsilon^{-1 / 2}+T(\varepsilon)\right]$.

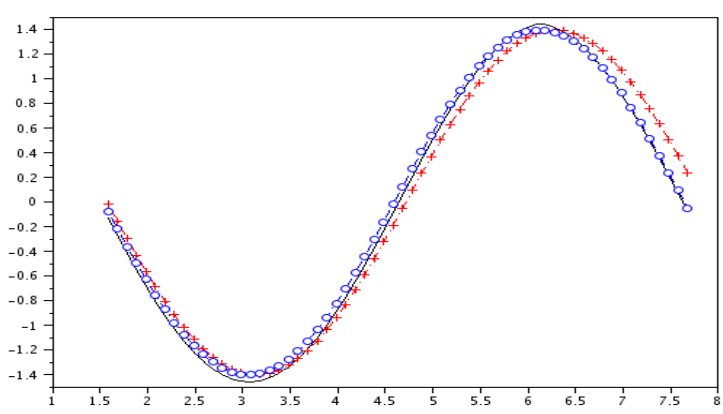

Fig. $5 \varepsilon=0.4$, time interval $\left[\varepsilon^{-1 / 2}, \varepsilon^{-1 / 2}+T(\varepsilon)\right]$.

The improved solution is better than the strained coordinates approximation.

We now turn to the usual time $\frac{1}{\varepsilon}$ for the method of strained coordinates in Figures $6,7,8$. The strained coordinates method is far to reach the precision $\varepsilon^{2}$ in these cases.

\section{Conclusion and prospects}

To understand the accuracy of the Lindstedt-Poincaré method to compute nonlinear normal modes for a $\mathrm{N}$ 


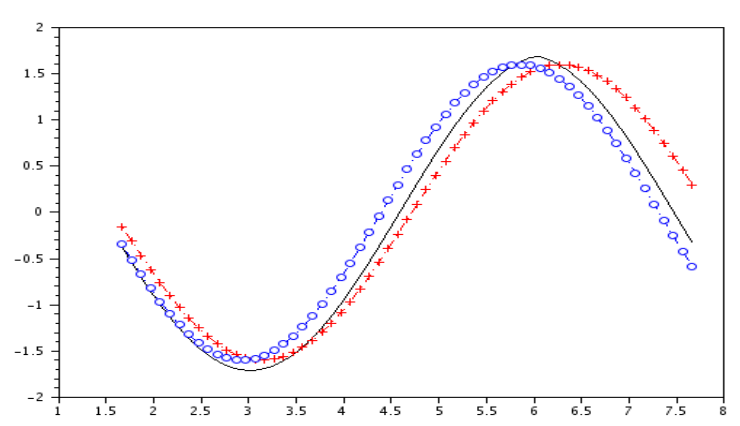

Fig. $6 \varepsilon=0.6$, time interval $\left[\varepsilon^{-1}, \varepsilon^{-1}+T(\varepsilon)\right]$.

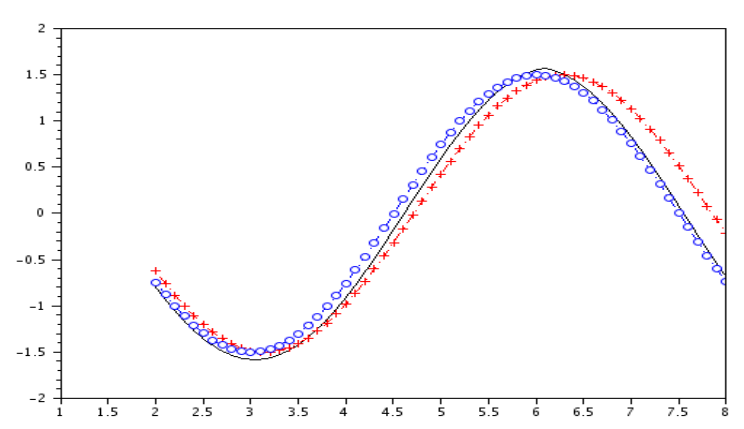

Fig. $7 \varepsilon=0.5$, time interval $\left[\varepsilon^{-1}, \varepsilon^{-1}+T(\varepsilon)\right]$.

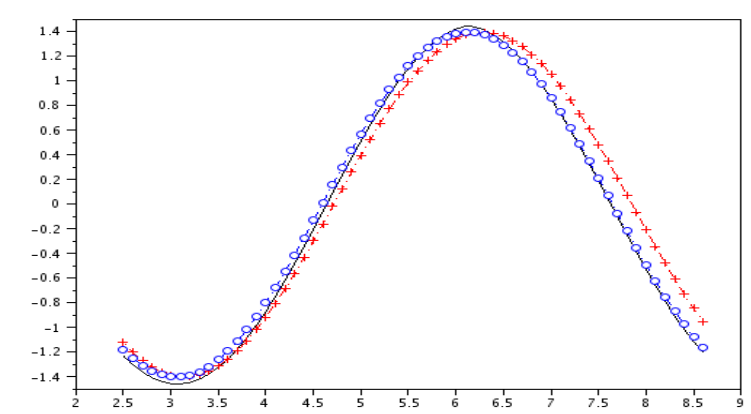

Fig. $8 \varepsilon=0.4$, time interval $\left[\varepsilon^{-1}, \varepsilon^{-1}+T(\varepsilon)\right]$.

d.o.f. piecewise linear problem, we have sharply studied the method of strained coordinates on a one d.o.f. problem with small grazing contact. This method is still working but there are some drawbacks compared to a smooth contact.

- The expansion is only the crude expansion of the linear problem. Nonlinear phenomenons are missed.

- The accuracy of the period is spoiled.

- The expansion of the period is wrong at the third order.T $(\varepsilon)$

- The time validity of the expansion is smaller.
Naturally, the grazing contact is a very weak contact, so the method has to be more precise to catch nonlinear phenomenons. These drawbacks suggest some improvements.

8.1 Improvements and open problem for grazing nonlinear modes

The usual method of strained coordinates has to be modified to compute nonlinear grazing modes for piecewiselinear systems. Our study proves rigorously that the right ansatz for the period and the pulsation with one d.o.f. is:

$T(\varepsilon)=T_{0}+\varepsilon^{2.5} T_{2.5}^{*}+\mathcal{O}\left(\varepsilon^{3.5}\right)$,
$\omega(\varepsilon)=\omega_{0}+\varepsilon^{2.5} \omega_{2.5}^{*}+\mathcal{O}\left(\varepsilon^{3.5}\right)$.

Using the more precise pulsation $\omega_{\varepsilon}^{*}=\omega_{0}+\omega_{2.5}^{*} \varepsilon^{2.5}$ combined with the linear approximation $v_{\varepsilon}(t)$ we have already a better approximation of the exact solution $u_{\varepsilon}(t)$ with

$$
v_{\varepsilon}\left(\omega_{\varepsilon}^{*} t\right)
$$

than the strained coordinates approximation. How to compute $\omega_{2.5}^{*}$ in general? It is not clear with the generalized Taylor expansion (16) used in [4] because the fractional powers do not appear explicitly. Could we improve the profile $v_{\varepsilon}$ ? It is less clear as Theorem (4.2) in $[4]$.

In conclusion the limitation of the method of strained coordinates is proved precisely but the improvment of this method has to be discovered.

\section{Appendix 1}

In this Appendix we compute the following exact expression for the period with the energy.

$$
\begin{array}{r}
T=\left\{\begin{array}{r}
2 \pi-2 \arccos \left(\left|u_{-}\right|^{-1}\right) \\
+2 \sqrt{\eta} \arccos \left(\frac{\eta}{h_{0}+\eta}\right)
\end{array}\right. \\
u_{-}=-\sqrt{E_{0}}, \quad \eta=\frac{1}{1+\varepsilon} .
\end{array}
$$

$T(\varepsilon)$ To obtain this period formula, i.e. Formula (11) before, we use the well known relation between the energy and the period [7]. Using the symmetry with respect to the horizontal axis in the phase space, we compute the half period:

$$
\frac{T}{2}=\int_{u_{-}}^{u_{0}} \frac{d u}{\sqrt{E_{0}-F(u)}} .
$$

Formula (28) comes from the relation $\dot{u}= \pm \sqrt{E-F(u)}$. For the half superior part: $\dot{u}>0$ and since $E=E_{0}$, the 
relation becomes: $\frac{d u}{d t}=\sqrt{E_{0}-F(u)}$, so $d t=\frac{d u}{\sqrt{E_{0}-F(u)}}$ which yields relation (28).

Since $F(u)$ has different expression for $u<1$ or $u>1$ we decompose the integral in (28) in two parts.. The computation is explicit with the function arcsin and the following relations for $A>0$ :

$$
\arcsin (y)=\int_{0}^{y} \frac{d u}{\sqrt{1-u^{2}}},
$$

$\arcsin \left(\frac{y+b}{\sqrt{A+b^{2}}}\right)=\int^{y} \frac{d u}{\sqrt{A-\left(u^{2}+2 b u\right)}}$.

Equality (30) means the left hand side is an antiderivative of the right hand side. We also recall the arccos function for $y \in[0,1]$ :

$\arccos (y)=\frac{\pi}{2}-\arcsin (y)=\int_{y}^{1} \frac{d u}{\sqrt{1-u^{2}}}$.

Now we can compute $T / 2$ :

$\frac{T}{2}=T_{-}+T_{0}=\int_{u_{-}}^{1} \frac{d u}{\sqrt{E_{0}-F(u)}}+\int_{1}^{u_{0}} \frac{d u}{\sqrt{E_{0}-F(u)}}$.

Notice that $u_{-}=-\sqrt{E_{0}}$ so:

$$
\begin{aligned}
T_{-} & =\int_{u_{-}}^{1} \frac{d u}{\sqrt{E_{0}-u^{2}}} \\
& =\arcsin \left(1 / \sqrt{E_{0}}\right)-\arcsin \left(u_{-} / \sqrt{E_{0}}\right), \\
& =-\arcsin \left(1 / u_{-}\right)-\arcsin (-1)=\frac{\pi}{2}+\arcsin \left(1 /\left|u_{-}\right|\right), \\
& =\pi-\arccos \left(1 /\left|u_{-}\right|\right) .
\end{aligned}
$$

We now turn to $T_{0}$ with the notation $u=1+v$ and $\eta=1 /(1+\varepsilon)$ :

$$
\begin{aligned}
T_{0}= & \int_{1}^{u_{0}} \frac{d u}{\sqrt{E_{0}-\left(u^{2}+\varepsilon v^{2}\right)}}, \\
= & \int_{0}^{h_{0}} \frac{d v}{\sqrt{\left(1+h_{0}\right)^{2}+\varepsilon h_{0}^{2}-\left((1+v)^{2}+\varepsilon v^{2}\right)}}, \\
= & \int_{0}^{h_{0}} \frac{d v}{\sqrt{(1+\varepsilon)\left(h_{0}^{2}-v^{2}\right)+2\left(h_{0}-v\right)}}, \\
= & \sqrt{\eta} \int_{0}^{h_{0}} \frac{d v}{\sqrt{\left(h_{0}^{2}-v^{2}\right)+2 \eta\left(h_{0}-v\right)}}, \\
= & \sqrt{\eta} \int_{0}^{h_{0}} \frac{d v}{\sqrt{\left(h_{0}^{2}+2 \eta h_{0}\right)-\left(v^{2}+2 \eta v\right)}}, \\
= & \sqrt{\eta}\left(\arcsin \left(\frac{h_{0}+\eta}{h_{0}+\eta}\right)-\arcsin \left(\frac{\eta}{h_{0}+\eta}\right)\right) \\
& \text { from }(30) \\
& \operatorname{and} A+b^{2}=h_{0}^{2}+2 \eta h_{0}+\eta^{2}=\left(h_{0}+\eta\right)^{2}, \\
= & \sqrt{\eta}\left(\frac{\pi}{2}-\arcsin \left(\frac{\eta}{h_{0}+\eta}\right)\right) \\
= & \sqrt{\eta} \arccos \left(\frac{\eta}{h_{0}+\eta}\right) .
\end{aligned}
$$

Adding $T_{-}$and $T_{0}$ we obtain $T / 2$ and then (11).

\section{Appendix 2}

We compute (26): the asymptotic expansion of the period $T(\varepsilon)$ from the exact expression of the period (11). $T(\varepsilon)=2 \pi-\frac{7}{6} \sqrt{2} \varepsilon^{2.5}+\mathcal{O}\left(\varepsilon^{3.5}\right)$.

The details of the computations first use an expansion of the function $\arccos (y)$ near $y=1$.

$\arccos (1-h)=\left\{\begin{array}{l}\sqrt{2 h}+\frac{\sqrt{2}}{12} h^{1.5}+\frac{3 \sqrt{2}}{80} h^{2.5} \\ +\mathcal{O}\left(h^{3.5}\right) .\end{array}\right.$

We now prove this formula. First, by (31) we have:

$$
\begin{aligned}
\arccos (1-h)= & \int_{1-h}^{1} \frac{d u}{\sqrt{1-u^{2}}}, \\
= & \int_{1-h}^{1} \frac{d u}{\sqrt{(1-u)(1+u)}}, \\
& \text { with } u=1-t h \text { we have: } \\
= & \int_{0}^{1} \frac{h d t}{\sqrt{t h(2-t h)}}, \\
= & \sqrt{h} \int_{0}^{1} \frac{d t}{\sqrt{t(2-t h)}}, \\
= & \sqrt{h} g(h) .
\end{aligned}
$$

We now have an integral with the parameter $h$ which is not singular. Thus $g$ is a smooth function and we have the following expansion with fractional powers of $h$ :

$$
\begin{gathered}
\arccos (1-h) \\
=\sqrt{h}\left[g(0)+g^{\prime}(0) h+\frac{g^{\prime \prime}(0)}{2} h^{2}+\mathcal{O}\left(h^{3}\right)\right], \\
=g(0) \sqrt{h}+g^{\prime}(0) h^{1.5}+\frac{g^{\prime \prime}(0)}{2} h^{2.5}+\mathcal{O}\left(h^{3.5}\right) .
\end{gathered}
$$

We compute $g(0), g^{\prime}(0)$ and $g^{\prime \prime}(0)$ to get formula (32).

$$
\begin{aligned}
g(h) & =\int_{0}^{1} \frac{d t}{\sqrt{t(2-t h)}}, \\
g(0) & =\int_{0}^{1} \frac{d t}{\sqrt{2 t}}=\sqrt{2}, \\
g^{\prime}(h) & =\frac{1}{2} \int_{0}^{1} \frac{\sqrt{t} d t}{(2-t h)^{3 / 2}}, \\
g^{\prime}(0) & =\frac{1}{2^{5 / 2}} \int_{0}^{1} \sqrt{t} d t=\frac{1}{6 \sqrt{2}}=\frac{\sqrt{2}}{12}, \\
g^{\prime \prime}(h) & =\frac{3}{4} \int_{0}^{1} \frac{t \sqrt{t} d t}{(2-t h)^{5 / 2}}, \\
g^{\prime \prime}(0) & =\frac{3}{2^{9 / 2}} \int_{0}^{1} t^{3 / 2} d t=\frac{3 \sqrt{2}}{80} .
\end{aligned}
$$

We now turn to asymptotic expansion of the exact period $T(\varepsilon)$. For this purpose, we compute the expansions of the two last terms defining the exact period 
$T(\varepsilon)=2 \pi+T_{2}+T_{3}$ defined by (11):

$T_{2}=-2 \arccos \left(\left|u_{-}\right|^{-1}\right)$,

$T_{3}=2 \sqrt{\eta} \arccos \left(\frac{\eta}{\varepsilon+\eta}\right)$

To expand $T_{2}$ with respect to $\varepsilon$, we notice that from (28), (25):

$$
\begin{aligned}
\left|u_{-}\right| & =\sqrt{(1+\varepsilon)^{2}+\varepsilon^{3}}, \\
& =(1+\varepsilon) \sqrt{1+\frac{\varepsilon^{3}}{(1+\varepsilon)^{2}}}, \\
& =(1+\varepsilon)\left(1+\frac{\varepsilon^{3}}{2}+\mathcal{O}\left(\varepsilon^{4}\right)\right), \\
& =1+\varepsilon+\frac{\varepsilon^{3}}{2}+\mathcal{O}\left(\varepsilon^{4}\right), \\
\frac{1}{\left|u_{-}\right|} & =1-\varepsilon+\varepsilon^{2}-\frac{\varepsilon^{3}}{2}+\mathcal{O}\left(\varepsilon^{4}\right) .
\end{aligned}
$$

Let $h$ be defined as:

$$
\begin{aligned}
h & =1-\left|u_{-}\right|^{-1}=\varepsilon-\varepsilon^{2}+\frac{\varepsilon^{3}}{2}+\mathcal{O}\left(\varepsilon^{4}\right), \\
\sqrt{h} & =\sqrt{\varepsilon} \sqrt{1-\varepsilon+\frac{\varepsilon^{2}}{2}+\mathcal{O}\left(\varepsilon^{3}\right),} \\
& =\sqrt{\varepsilon}\left(1-\frac{\varepsilon}{2}+\frac{\varepsilon^{2}}{4}+\frac{3}{8} \varepsilon^{2}+\mathcal{O}\left(\varepsilon^{3}\right)\right), \\
& =\sqrt{\varepsilon}-\frac{\varepsilon^{1.5}}{2}+\frac{5}{8} \varepsilon^{2.5}+\mathcal{O}\left(\varepsilon^{3.5}\right), \\
h^{1.5} & =\varepsilon^{1.5}-\frac{3}{2} \varepsilon^{2.5}+\mathcal{O}\left(\varepsilon^{3.5}\right), \\
h^{2.5} & =\varepsilon^{2.5}+\mathcal{O}\left(\varepsilon^{3.5}\right) .
\end{aligned}
$$

We now can compute $T_{2}$ :

$$
\begin{aligned}
-T_{2} & =2 \arccos (1-h), \\
& \left.=2 \sqrt{2 h}+\frac{\sqrt{2}}{6} h^{1.5}+\frac{3 \sqrt{2}}{80} h^{2.5}+\mathcal{O}\left(h^{3.5}\right)\right), \\
& \left.=2 \sqrt{2} \sqrt{\varepsilon}-\frac{5 \sqrt{2}}{6} \varepsilon^{1.5}+\frac{83 \sqrt{2}}{80} \varepsilon^{2.5}+\mathcal{O}\left(\varepsilon^{3.5}\right)\right) .
\end{aligned}
$$

For the third term, we have

$$
\begin{aligned}
\frac{\eta}{\varepsilon+\eta} & =\left(1+\varepsilon+\varepsilon^{2}\right)^{-1} \\
& =1-\varepsilon+\varepsilon^{3}+\mathcal{O}\left(\varepsilon^{4}\right)=1-h .
\end{aligned}
$$

Now we expand $h, h^{1.5}$ and $h^{2.5}$ :

$$
\begin{aligned}
h & =\varepsilon-\varepsilon^{3}+\mathcal{O}\left(\varepsilon^{4}\right)=\varepsilon\left(1-\varepsilon^{2}+\mathcal{O}\left(\varepsilon^{3}\right)\right), \\
\sqrt{h} & =\sqrt{\varepsilon}-\frac{\varepsilon^{2.5}}{2}+\mathcal{O}\left(\varepsilon^{3.5}\right), \\
h^{1.5} & =\varepsilon^{1.5}+\mathcal{O}\left(\varepsilon^{3.5}\right), \\
h^{2.5} & =\varepsilon^{2.5}+\mathcal{O}\left(\varepsilon^{3.5}\right),
\end{aligned}
$$

These yields to the expansion of $T_{3}$ :

$$
\begin{aligned}
& 2 \arccos \left(\frac{\eta}{\varepsilon+\eta}\right)= 2 \sqrt{2} \sqrt{\varepsilon}+\frac{\sqrt{2}}{6} \varepsilon^{1.5} \\
&\left.-\frac{191 \sqrt{2}}{240} \varepsilon^{2.5}+\mathcal{O}\left(\varepsilon^{3.5}\right)\right), \\
& \sqrt{\eta}=(1+\varepsilon)^{-1 / 2}= 1-\frac{\varepsilon}{2}+\frac{3}{8} \varepsilon^{2}+\mathcal{O}\left(\varepsilon^{3}\right), \\
&\left.T_{3}=2 \sqrt{2} \sqrt{\varepsilon}-\frac{5 \sqrt{2}}{6} \varepsilon^{1.5}-\frac{31 \sqrt{2}}{240} \varepsilon^{2.5}+\mathcal{O}\left(\varepsilon^{3.5}\right)\right) .
\end{aligned}
$$

Finally, adding the expansions for $T_{2}$ and $T_{3}$ we obtain

$$
\left.T-2 \pi=-\frac{280 \sqrt{2}}{240} \varepsilon^{2.5}+\mathcal{O}\left(\varepsilon^{3.5}\right)\right),
$$

and then (26).

\section{Appendix 3}

We compute the exact solution to compare it numerically with some asymptotic expansions. Since the ODE (7) is piecewise linear, we have after some computations:

$$
\begin{array}{lr}
u_{\varepsilon}(t)=\eta \varepsilon+\left(1+\eta \varepsilon^{2}\right) \cos (\sqrt{1+\varepsilon} t), & 0<t<\tau, \\
u_{\varepsilon}(t)=u_{-} \cos \left(t-\frac{T}{2}\right), & \tau<t<T-\tau, \\
u_{\varepsilon}(t)=u_{\varepsilon}(T-t), & T-\tau<t<T,
\end{array}
$$

where $u_{-}$and $\eta$ are defined in section $3, T=T(\varepsilon)$ and

$$
\tau=\tau(\varepsilon)=\frac{\arccos \left(\frac{1-\eta \varepsilon}{1+\eta \varepsilon^{2}}\right)}{\sqrt{1+\varepsilon}} .
$$

Acknowledgements We thank Claude-Henri Lamarque, Mathias Legrand and Bernard Rousselet for fruitful discussions on nonlinear normal modes.

\section{References}

1. D. Jiang, C. Pierre, and S.W. Shaw. Large-amplitude non-linear normal mode of piecewise linear systems. $J$. Sound. Vibration., 272:869-891, 2004.

2. G. Kerschen, M. Peeters, J.C. Golinval, and A.F. Vakakis. Nonlinear normal modes, part i: A useful framework for the structural dynamics. Mechanical Systems and Signal Processing, 23:170-194, 2009.

3. F. Vestroni, A. Luongo, and A. Paolone. A perturbation method for evaluating nonlinear normal modes of a piecewise linear two-degrees-of-freedom system. Nonlinear Dynam., 54(4):379-393, 2008.

4. S. Junca and B. Rousselet. The method of strained coordinates for vibrations with weak unilateral springs. IMA J. Appl. Math., 76(2):251-276, 2010.

5. J. Kevorkian and J.D. Cole. Perturbation methods in applied mathematics. Number 34 in Applied Mathematical Sciences. Springer-Verlag, New York-Berlin, 1981. 
6. J. Kevorkian and J.D. Cole. Multiple Scale and Singular Perturbations Problems. Number 114 in Applied Mathematical Sciences. Springer-Verlag, New York-Berlin, 1996.

7. P.D. Miller. Applied Asymptotic Analysis. Number 75. American Mathematical Society, Providence, Rhode Island, 1996.

8. S. Junca and B. Rousselet. Asymptotic Expansions of Vibrations with Small Unilateral Contact, pages 173-182. Number 128. Springer proceedings in physics, 2009.

9. A. Luongo and D. Zulli. A paradigmatic system to study the transition from zero/hopf to double-zero/hopf bifurcation. Nonlinear Dynam., 70(1):111-124, 2012.

10. J. Bastien, F. Bernardin, and C.-H. Lamarque. Nonsmooth deterministic or stochastic discrete dynamical systems [Applications to models with friction or impacts]. Mechanical engineering and solid mechanics series. Wiley, 2013.

11. A.H. Nayfeh. Perturbation methods. Pure and Applied Mathematics. John Wiley and Sons, New York-LondonSydney, 1973. 